\title{
The Study of Heat Generation and Viscous Dissipation on Mhd Heat And Mass Diffusion Flow Past A Surface
}

\author{
K. Bhagya Lakshmi ${ }^{1}$, G.S.S. Raju ${ }^{2}$, P.M. Kishore ${ }^{3}$ and N.V.R.V.Prasada Rao ${ }^{4}$ \\ 1. Department of Mathematics, C.M.R. Technical Campus, Kandlakoya (V), Medchal (M), Hyderabad-501401. \\ (A.P), India. \\ 2. Department of Mahtematics, J.N.T.U. A. College of Engineering, Pulivendula, Y.S.R. District. (A.P), India. \\ 3. Department of Mathematics, Narayana Engineering College, Nellore - 524001 (A.P), India. \\ 4. Department of Mathematics, S.V.G.S. College, Nellore - 524002 (A.P), India.
}

\begin{abstract}
The present work is devoted to the numerical study of magneto hydrodynamic (MHD) natural convection flow of heat and mass transfer past a plate taking into account viscous dissipation and internal heat generation. The governing equations and the associated boundary conditions for this analysis are made non dimensional forms using a set of dimensionless variables. Thus, the non dimensional governing equations are solved numerically using finite difference method Crank-Nicolson's scheme. Numerical outcomes are found for different values of the magnetic parameter, Modified Grashof number, Prandtl number, Eckert number, heat generation parameter and Schmidt number for the velocity and the temperature within the boundary layer as well as the skin friction coefficients and the rate of heat and mass transfer along the surface. Results are presented graphically with detailed discussion.
\end{abstract}

Key Words: MHD, heat generation, free convection, viscous dissipation, finite difference method.

\section{Introduction}

The buoyancy force induced by density differences in a fluid causes natural convection. Natural convection flows are frequently encountered in physical and engineering problems such as chemical catalytic reactors, nuclear waste materials etc. Transient free convection is important in many practical applications, such as furnaces electronic components, solar collectors, thermal regulation process, security of energy systems etc. when a conductive fluid moves through a magnetic field and an ionized gas is electrically conductive, the fluid may be influenced by the magnetic field. Magnetohydrodynamic free convection heat transfer flow is of considerable interest in the technical field due to its frequent occurrence in industrial technology and geothermal application, liquid metal fluids and MHD power generation systems etc. The change in wall temperature causing the free convection flow could be a sudden or a periodic one, leading to a variation in the flow. In nuclear engineering, cooling of medium is more important safety point of view and during this cooling process the plate temperature starts oscillating about a non-zero constant mean temperature. Further, oscillatory flow has applications in industrial and aerospace engineering. Viscous mechanical dissipation effects are important in geophysical flows and also in certain industrial operations and are usually characterized by the Eckert number. Many practical heat transfer applications involve the conversion of some form of mechanical, electrical, nuclear, or chemical energy to thermal energy in the medium. Such mediums are said to involve internal heat generation. For example, a large amount of heat is generated in the fuel elements from atomic reactors as a result of atomic fission that serves as the heat source for the nuclear power plants. The heat generated in the sun as a result of fusion of hydrogen into helium makes the sun a large nuclear reactor that supplies heat to the earth. Possible heat generation effects may modify temperature distribution and, therefore, the particle deposition rate.

In the literature, extensive research work is available to examine the effect of natural convection on flow past a plate. Examples of this include Vedhanayagam et.al. [1], Kolar et. al. [2] and Li et. al. [3]. Transient free convection flow past an isothermal vertical plate was first reported by Siegel [4] using an integral method. The experimental confirmation of these results was discussed by Goldstein et. al. [5]. A review of transient natural convection presented by Raithby et.al [6] wherein a large number of papers on this topic were reviewed. In this review, the meaning of transient convection has been explained systematically. They have defined the conduction regime and the steady state regime and that which lies between these two regimes as the transient regime. Other studies dealing with transient natural convection are by Das et.al. [7] and Saeid [8]. Simultaneous heat and mass transfer in laminar free convection boundary layer flows over surface can be found in Lin et al. [9] and Mongruel et.al. [10].

Fewer studies have been carried out to investigate the magnetohydrodynamic free convection flow in the presence of viscous dissipation. In all the investigations mentioned above, viscous mechanical dissipation is neglected. A number of authors have considered viscous heating effects on Newtonian flows. Isreal-Cookey et.al. [11] investigated the influence of viscous dissipation and radiation on unsteady MHD free convection flow 
past an infinite heated vertical plate in a porous medium with time dependent suction. Zueco [12] used network simulation method (NSM) to study the effects of viscous dissipation and radiation on unsteady MHD free convection flow past a vertical porous plate. Suneetha et.al. [13] have analyzed the thermal radiation effects on hydromagnetic free convection flow past an impulsively started vertical plate with variable surface temperature and concentration is analyzed by taking into account of the heat due to viscous dissipation. Recently Hiteesh [14] studied the boundary layer steady flow and heat transfer of a viscous incompressible fluid due to a stretching plate with viscous dissipation effect in the presence of a transverse magnetic field.

The heat transfer in a laminar boundary layer flow of a viscous fluid over a linearly stretching continuous surface with viscous dissipation/frictional heating and internal heat generation was analysed by Vajravelu and Hadjinicolaou [15]. They considered the volumetric rate of heat generation, $q$ "'[$\left[\mathrm{W} / \mathrm{m}^{3}\right]$, as $q^{\prime \prime}\left[W / m^{3}\right]=Q_{0}\left(T_{f}-T_{\infty}\right)$, for $T_{f} \geq T_{\infty}$ and, $q^{\prime \prime \prime}=0$, for $T_{f} \geq T_{\infty}$, where $Q_{0}$ is the heat generation constant. Madhusudhana Rao et.al. [16] studied the analysis of unsteady free convection heat and mass transfer flow through a non-homogeneous porous medium with variable permeability bounded by an infinite porous vertical plate in slip flow regime taking into account the radiation, chemical reaction and temperature gradient dependent heat source. Mamun et al. [17] investigated MHD-conjugate heat transfer analysis for a vertical flat plate in presence of viscous dissipation and heat generation. Recently, Azim et al. [18] analyzed viscous Joule heating MHD-conjugate heat transfer for a vertical flat plate in the presence of heat generation.

Hence, based on the above mentioned investigations and applications, the object of this paper is to study magnetohydrodynamic transient heat and mass transfer flow by free convection past a vertical plate in the presence of viscous dissipation and heat generation, when the temperature of the plate oscillates periodically about a constant mean temperature. The present investigation may be useful for the study of movement of oil or gas and water through the reservoir of an oil or gas field, underground water in river beds, filtration and water purification processes. This study of flow past a vertical surface can be utilized as the basis of many scientific and engineering applications, including earth science, nuclear engineering and metallurgy. In nuclear engineering, it finds its applications for the design of the blanket of liquid metal around a thermonuclear fusionfission hybrid reactor. In metallurgy, it can be applied during the solidification process. The results of the problem are also of great interest in geophysics, in the study of interaction of geomagnetic field with the fluid in the geothermal region.

\section{Mathematical Analysis}

We consider a one - dimensional flow of an incompressible and electrically conducting viscous fluid along an infinite vertical plate. The $x^{\prime}$-axis is taken along the infinite plate and $y^{\prime}$ - axis normal to it. Initially, the plate and the fluid are at same temperature $T_{\infty}^{\prime}$ with concentration level $C_{\infty}^{\prime}$ at all points. At time $t^{\prime}>0$, the plate temperature is raised to $T_{w}^{\prime}$ and a periodic temperature variation is assumed to be superimposed on this mean constant temperature of the plate and the concentration level at the plate is raised to $C_{w}^{\prime}$. A magnetic field of uniform strength is applied perpendicular to the plate and the magnetic Reynolds number is assumed to be small so that the induced magnetic field is neglected [19]. There is no applied electric field. The MHD term is derived from an order-of-magnitude analysis of the full Navier-Stokes equations. Under these conditions and assuming variation of density in the body force term (Boussinesq's approximation), the problem can be governed by the following set of equations:

$$
\begin{aligned}
& \frac{\partial u^{\prime}}{\partial t^{\prime}}=v \frac{\partial^{2} u^{\prime}}{\partial y^{\prime 2}}+\left[g \beta\left(T^{\prime}-T_{\infty}^{\prime}\right)+g \beta_{c}\left(C^{\prime}-C_{\infty}^{\prime}\right]-\frac{\sigma B_{0}^{2} u^{\prime}}{\rho}\right. \\
& \frac{\partial T^{\prime}}{\partial t^{\prime}}=\frac{k}{\rho c_{p}} \frac{\partial^{2} T^{\prime}}{\partial y^{\prime 2}}+\frac{v}{\rho c_{p}}\left(\frac{\partial u^{\prime}}{\partial y^{\prime}}\right)^{2}+\frac{Q^{\prime}}{\rho c_{p}}\left(T^{\prime}-T_{\infty}^{\prime}\right) \\
& \frac{\partial C^{\prime}}{\partial t^{\prime}}=D \frac{\partial^{2} C^{\prime}}{\partial y^{\prime 2}}
\end{aligned}
$$

with the following initial and boundary conditions: 
$u^{\prime}=0, \quad T^{\prime}=T_{\infty}^{\prime}, \quad C^{\prime}=C_{\infty}^{\prime} \quad$ for all $y^{\prime}, t^{\prime} \leq 0$

$u^{\prime}=0, \quad T^{\prime}=T_{w}^{\prime}+\in\left(T_{w}^{\prime}-T_{\infty}^{\prime}\right) \cos w^{\prime} t^{\prime}, C^{\prime}=C_{w}^{\prime}$ at $y^{\prime}=0, t^{\prime}>0$

$u^{\prime} \rightarrow 0, \quad T^{\prime} \rightarrow T_{\infty}^{\prime}, \quad C^{\prime} \rightarrow C_{\infty}^{\prime} \quad$ as $\quad y^{\prime} \rightarrow \infty, t^{\prime}>0$

The second term of R.H.S. of the momentum equation (1) denotes buoyancy effects, the third term is the MHD term. The second term of R.H.S. of the energy equation (2) denotes radiation term, the third term is viscous dissipation term. The heat generation and heat due to viscous dissipation is taken into an account in equation (2).

The temperature distribution is independent of the flow and heat transfer is by conduction alone. This is true for fluids in initial stage due to the absence of convective heat transfer or at small Grashof number flow ( $\mathrm{Gr}$ $\leq 1)$.

Skin - friction is given by $\tau_{s}^{\prime}=-\mu\left(\frac{\partial u^{\prime}}{\partial y^{\prime}}\right)_{y=0}$

We introduce the non-dimensional variables:

$t=\frac{t^{\prime}}{t_{R}}, \quad y=\frac{y^{\prime}}{L_{R}}, \quad u=\frac{u^{\prime}}{U_{R}}, \quad w=w^{\prime} t_{R}, \quad \operatorname{Pr}=\frac{\mu C_{p}}{k}, \quad Q=\frac{Q^{\prime} L_{R}^{2}}{\rho c_{p} v}$

$M=\frac{\sigma B_{0}^{2} v}{\rho U_{R}^{2}}, \quad S c=\frac{v}{D}, \quad \theta=\frac{T^{\prime}-T_{\infty}^{\prime}}{T_{w}^{\prime}-T_{\infty}^{\prime}}, \quad C=\frac{C^{\prime}-C_{\infty}^{\prime}}{C_{w}^{\prime}-C_{\infty}^{\prime}}, \quad \Delta T=T_{w}^{\prime}-T_{\infty}^{\prime}$,

$G m=\frac{v g \beta_{c}\left(C_{w}^{\prime}-C_{\infty}^{\prime}\right)}{U_{R}^{3}}, \quad U_{R}=(v g \beta \Delta T)^{1 / 3}, \quad L_{R}=\left(\frac{g \beta \Delta T}{v^{2}}\right)^{-1 / 3}$,

$t_{R}=(g \beta \Delta T)^{-2 / 3} v^{1 / 3}, \quad E=\frac{U_{R}^{2}}{C_{P} \Delta T}$

The equations (1) - (3) reduce to following non-dimensional form:

$\frac{\partial u}{\partial t}=\frac{\partial^{2} u}{\partial y^{2}}+\theta+G m C-M u$

$\frac{\partial \theta}{\partial t}=\frac{1}{\operatorname{Pr}} \frac{\partial^{2} \theta}{\partial y^{2}}+E\left(\frac{\partial u}{\partial y}\right)^{2}+Q \theta$

Sc $\frac{\partial C}{\partial t}=\frac{\partial^{2} C}{\partial y^{2}}$

with the following initial and boundary conditions:
$u=0$,
$\theta=0$
$C=0$
for all $y, t \leq 0$
$u=0$,
$\theta=1+\in \cos \omega t$
$C=1$
at $y=0, t>0$
$u \rightarrow 0$,
$\theta \rightarrow 0$
$C \rightarrow 0$
as $y \rightarrow \infty, t>0$

where $\omega t$ is phase angle.

Skin - Friction: In non-dimensional form, the skin - friction is given by 


$$
\tau=-\left(\frac{\partial u}{\partial y}\right)_{y=0}
$$

Nusselt Number: From temperature field, the rate of heat transfer in non-dimensional form is expressed as

$$
N u=-\left(\frac{\partial \theta}{\partial y}\right)_{y=0}
$$

Sherwood Number: From concentration field, the rate of mass transfer in non-dimensional form is expressed as

$$
S h=-\left(\frac{\partial C}{\partial y}\right)_{y=0}
$$

All the physical variables are defined in the nomenclature.

\section{Numerical Technique}

Equations $(2-7)-(2-9)$ are coupled non-linear partial differential equations and are to be solved under the initial and boundary conditions of equations (10) - (11). However exact or approximate solutions are not possible for this set of equations and hence we solve these equations by the implicit finite difference method of Crank - Nicolson's type. The finite difference equations corresponding to equations (7) - (9) are as follows:

$$
\begin{aligned}
&\left(\frac{u_{i, j+1}-u_{i, j}}{\Delta t}\right)=\left(\frac{u_{i-1, j+1}-2 u_{i, j+1}+u_{i+1, j+1}+u_{i-1, j}-2 u_{i, j}+u_{i+1, j}}{2(\Delta y)^{2}}\right) \\
&+\frac{1}{2}\left(\theta_{i, j+1}+\theta_{i, j}\right)+\frac{G m}{2}\left(C_{i, j+1}+C_{i, j}\right)-\frac{M}{2}\left(u_{i, j+1}+u_{i, j}\right) \\
& \frac{\theta_{i, j+1}-\theta_{i, j}}{\Delta t}= \frac{1}{\operatorname{Pr}}\left(\frac{\theta_{i-1, j+1}-2 \theta_{i, j+1}+\theta_{i+1, j+1}+\theta_{i-1, j}-2 \theta_{i, j}+\theta_{i+1, j}}{2(\Delta y)^{2}}\right) \\
&+\frac{Q}{2}\left(\theta_{i, j+1}+\theta_{i, j}\right)+E\left(\frac{u_{i+1, j}-u_{i, j}}{\Delta y}\right)^{2} \\
& S c\left(\frac{C_{i, j+1}-C_{i, j}}{\Delta t}\right)=\left(\frac{C_{i-1, j+1}-2 C_{i, j+1}+C_{i+1, j+1}+C_{i-1, j}-2 C_{i, j}+C_{i+1, j}}{2(\Delta y)^{2}}\right)
\end{aligned}
$$

Initial and boundary conditions take the following forms

$$
\begin{aligned}
& u_{i, 0}=0 \\
& \theta_{i, 0}=0, \\
& C_{i, 0}=0 \\
& \text { for all } i \\
& u_{0, j}=0 \text {, } \\
& \theta_{0, j}=1+\in \cos \omega t, \\
& C_{0, j}=1 \\
& u_{L, j}=0 \text {, } \\
& \theta_{L, j}=0 \text {, } \\
& C_{L, j}=0
\end{aligned}
$$

where $\mathrm{L}$ corresponds to $\infty$, the suffix ' $i$ ' corresponds to $y$ and ' $j$ ' corresponds to $t$.

Also $\Delta t=t_{j+1}-t_{j}$ and $\Delta y=y_{i+1}-y_{i}$.

Here we consider a rectangular grid with grid lines parallel to the coordinate axes with spacing $\Delta y$ and $\Delta t$ in space and time directions respectively. The grid points are given by $\mathrm{y}_{\mathrm{i}}=\mathrm{i} . \Delta \mathrm{y}, \mathrm{i}=1,2,3,---, \mathrm{L}-1$ and $\mathrm{t}_{\mathrm{j}}=\mathrm{j} . \Delta \mathrm{t}, \mathrm{j}$ $=1,2,3,---, P$. The spatial nodes on the $j^{\text {th }}$ time grid constitute the $\mathrm{j}^{\text {th }}$ layer or level. The maximum value of $y$ was chosen as 10 after some preliminary investigations, so that the two of the boundary conditions of equation (11) are satisfied. Here the maximum value of $y$ corresponds to $y=\infty$. After experimenting with few sets of mesh sizes, they have been fixed at the level $\Delta y=0.1$ and the time step $\Delta t=0.02$, in this case, special mesh size is reduced by $50 \%$ and the results are compared. It is observed that when mesh size is reduced by $50 \%$ in y direction, the result differ only in the fifth decimal place. 
The values of ' $\mathrm{C}, \theta$ and $\mathrm{u}$ ' are known at all grid points at $\mathrm{t}=0$ from the initial conditions. The values of ' $\mathrm{C}, \theta$ and $\mathrm{u}$ ' at time level ' $\mathrm{j}+1$ ' using the known values at previous time level ' $\mathrm{j}$ ' are calculated as follows. The finite difference equation (17) at every internal nodal point on a particular j- level constitutes a tri-diagonal system of equations. Such a system of equations is solved by using Thomas algorithm as discussed in Carnahan et al. [20]. Thus, the values of ' $\mathrm{C}$ ' are known at every nodal point at $(\mathrm{j}+1)^{\text {th }}$ time level. Similarly the values of ' $\theta$ ' are calculated from equation (16). Using the values of ' $C$ ' and ' $\theta$ ' at $(j+1)^{\text {th }}$ time level in equation (15), the values of ' $u$ ' at $(j+1)^{\text {th }}$ time level are found in similar manner. This process is continued to obtain the solution till desired time' $\mathrm{t}$ '. Thus the values of ' $\mathrm{C}, \theta$ and $\mathrm{u}$ ' are known, at all grid points in the rectangular region at the desired time level.

The local truncation error is $\mathrm{O}\left(\Delta t^{2}+\Delta y^{2}\right)$ and it tends to zero when ' $\Delta \mathrm{t}$ and $\Delta \mathrm{y}$ ' tend to zero. Hence the scheme is compatible. The finite difference scheme is unconditionally stable. Compatibility and stability ensures the convergence of the scheme.

The derivatives involved in equations (12) and (14) are evaluated using five point approximation formula.

\section{Results And Discussion}

The convection flows driven by combinations of diffusion effects are very important in many applications. The foregoing formulations may be analyzed to indicate the nature of interaction of the various contributions to buoyancy. In order to gain physical insight into the problem, the value of $\in$ is chosen 1.0. The values of Prandtl number are chosen $0.71,7$ which represent air and water respectively at $20^{\circ} \mathrm{c}$ temperature and 1 atmospheric pressure and the values of Schmidt number are chosen to represent the presence of species by hydrogen (0.22), water vapour (0.60), ammonia (0.78) and carbon dioxide $(0.96)$ at $25^{\circ} \mathrm{c}$ temperature and 1 atmospheric pressure.

Extensive computations were performed. Default values of the thermo physical parameters are specified as follows:

Magnetic parameter $M=2$, Modified Grashof number $G c=10$, Phase angle $\omega t=\frac{\pi}{2}$, Prandtl number $\operatorname{Pr}=$ 0.71 (air), Eckert number $E=0.5$, Heat generation parameter $\mathrm{Q}=0.5$, Schmidt number $S c=0.22$ (hydrogen) and time $t=0.2$. All graphs therefore correspond to these values unless otherwise indicated.

Fig (1) represents the velocity profiles due to the variations in $\omega t$. It is evident from the figure that the velocity increases and attains its maximum value in the vicinity of the plate and then tends to zero as $y \rightarrow \infty$. Moreover, the velocity is marginally affected by the variations in the phase angle. No reverse effect is observed in the case of heating of the surface $(\mathrm{Gm}<0)$.

Fig (2) reveals the effects of M, on the velocity profiles. It is obvious from the figure that the velocity near the plate exceeds i.e., the velocity overshoot occurs. It is observed that an increase in the value of ' $M$ ' leads to fall in the velocity. It is due to the application of transverse magnetic field will result a resistive type force (Lorentz force) similar to drag force which tends to resist the flow and thus reducing its velocity. The presence of a porous medium increases the resistance to flow resulting in decrease in the flow velocity. The opposite phenomenon is observed for heating of the plate $(\mathrm{Gm}<0)$.

The species concentration is coupled to the velocity via Modified Grashof number as seen in equation (7). For various values of Modified Grashof number, the velocity profiles are plotted in figure (3). It is obvious from the figure that the maximum velocity attains in the vicinity of the plate then decreases to zero as $y \rightarrow \infty$. It is observed that greater cooling of surface (an increase in $\mathrm{Gm}$ ) results in an increase in the velocity for air. It is due to the fact increase in the values of and mass Grashof number has the tendency to increase the mass buoyancy effect. This gives rise to an increase in the induced flow. The reverse effect is observed in case of $\mathrm{Gm}$ $<0$.

Fig. 4 shows the effect of variation of Prandtl number Pr. It is noticed that the velocity decreases as $\operatorname{Pr}$ increases. The velocity for $P r=0.71$ is higher than that of $P r=7$. Physically, it is possible because fluids with high Prandtl number have high viscosity and hence move slowly. It is also observed that no reverse effect for $\mathrm{Gm}<0$.

Effects of variations of time $t$ on the velocity are presented in fig. 5. It is clear from the fig that the velocity increases as the time $t$ increases and the reverse effect is noticed if $\mathrm{Gm}<0$.

For different values of the Schmidt number the velocity profiles are plotted in Fig. 6. It is obvious from the figure that an increase in the Schmidt number results in decrease in the velocity. It is observed that the reverse effect is obtained for $\mathrm{Gm}<0$.

Figure (7) reveals the transient temperature profiles against y (distance from the plate). The magnitude of temperature is maximum at the plate and then decays to zero asymptotically. The magnitude of temperature 
for air $(P r=0.71)$ is greater than that of water $(P r=7)$. This is due to the fact that thermal conductivity of fluid decreases with increasing ' $P r$ ', resulting a decrease in thermal boundary layer thickness. Also the temperature falls with an increase in the phase angle $\omega t$ for both air and water.

In figure (8) we depict the effect of Prandtl number (Pr) on the temperature field. It is observed that an increase in the Prandtl number leads to decrease in the temperature field. Also, temperature field falls more rapidly for water in comparison to air and the temperature curve is exactly linear for mercury $(\operatorname{Pr}=0.025)$, which is more sensible towards change in temperature. From this observation it is conclude that mercury is most effective for maintaining temperature differences and can be used efficiently in the laboratory. Air can replace mercury, the effectiveness of maintaining temperature changes are much less than mercury. However, air can be better and cheap replacement for industrial purpose. This is because, either increase of kinematic viscosity or decrease of thermal conductivity leads to increase in the value of Prandtl number (Pr). Hence temperature decreases with increasing of Prandtl number (Pr).

The effect of Eckert number ' $E$ ' on the temperature is shown in fig (9). Eckert number is the ratio of the kinetic energy of the flow to the boundary layer enthalpy difference. The effect of viscous dissipation on flow field is to increase the energy, yielding a greater fluid temperature and as a consequence greater buoyancy force. The increase in the buoyancy force due to an increase in the dissipation parameter enhances the temperature. It is also observed that the magnitude of temperature for air $(\operatorname{Pr}=0.71)$ is greater than that of water $(\operatorname{Pr}=7)$.

Effect of variations in $\mathrm{Q}$ on the temperature is presented in Fig. 10. It is clear from the figure that the temperature increases as heat generation parameter increases. It is also observed that the magnitude of temperature for air $(P r=0.71)$ is greater than that of water $(P r=7)$.

Fig (11) concerns with the effect of ' $t$ ' on the temperature. We see that temperature ' $\theta$ ' increases as time ' $t$ ' increases for both air $(\mathrm{Pr}=0.71)$ and water $(\mathrm{Pr}=7)$.

Fig (12) concerns with the effect of ' $S c$ ' on the concentration. It is noted that the concentration at all points in the flow field decreases exponentially with y and tends to zero as $y \rightarrow \infty$. A comparison of curves in the figure shows a decrease in concentration with an increase in Schmidt number. Physically it is true, since the increase of ' $S c$ ' means decrease of molecular diffusivity. That results in decrease of concentration boundary layer. Hence, the concentration of species is higher for small values of Sc and lower for large values of 'Sc'.

Fig (13) reveals the skin - friction against time $t$ for varies values of parameters $\mathrm{M}, \mathrm{Gm}, \mathrm{Sc}, \mathrm{E}, \omega t$ and Pr. It is noticed that the skin friction decreases with an increase in Eckert number, modified Grashof number and heat generator parameter while it increase with an increase in magnetic parameter, phase angle and Schmidt number for both air and water. The magnitude of the Skin-friction for water is greater than air and this behavior is reversed after ' $\mathrm{t}=0.8$ '.

Fig (14) depicts the Nusselt number against time' $t$ ' for various values of parameters ' $\omega t, \operatorname{Pr}, \mathrm{Q}, E$ and $\mathrm{Sc}^{\prime}$. It is found that the rate of heat transfer falls with increasing $\omega t$, Prandtl number, Eckert number, and heat generation parameter while it increases with an increase in Schmidt number.

It is marked from Fig. (15) that the rate of concentration transfer increases with increasing values of Schmidt number 'Sc' .

\section{Figures}

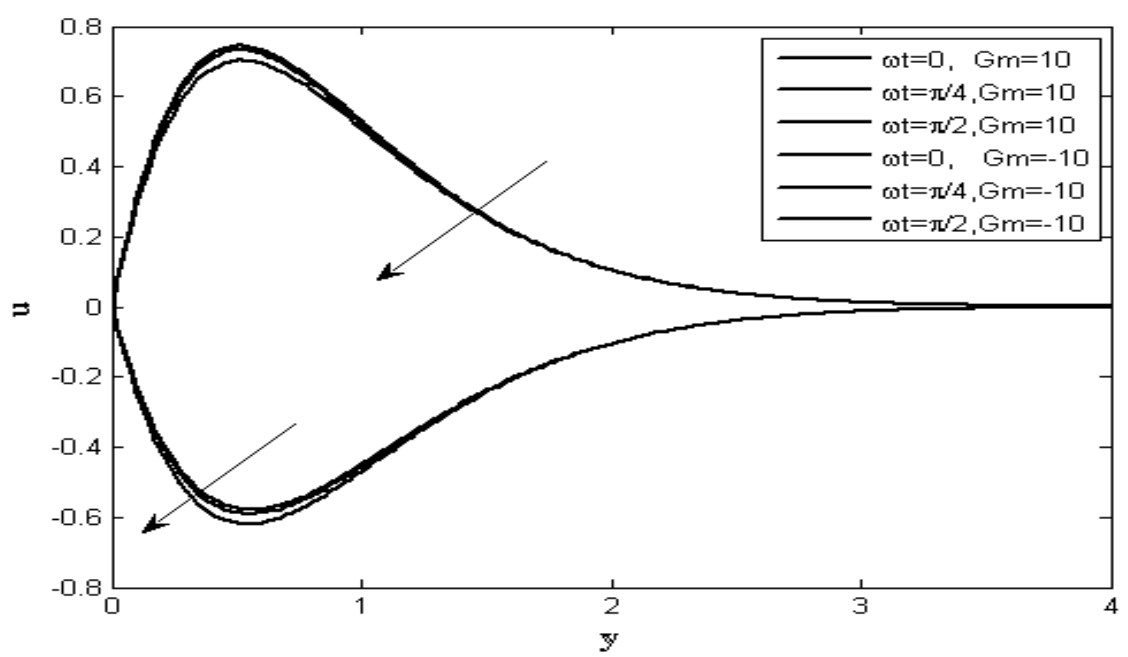

Fig.(1): Velocity profile for different values of $\omega t$ 


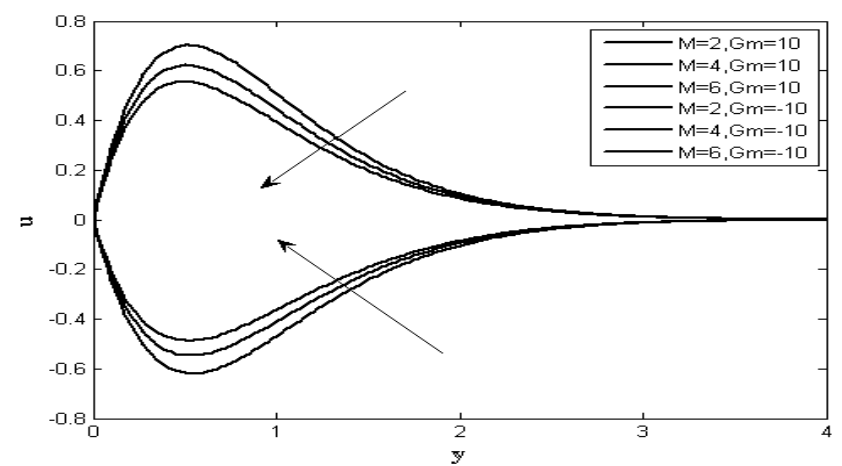

Fig.(2): Velocity profile for different values of $M$

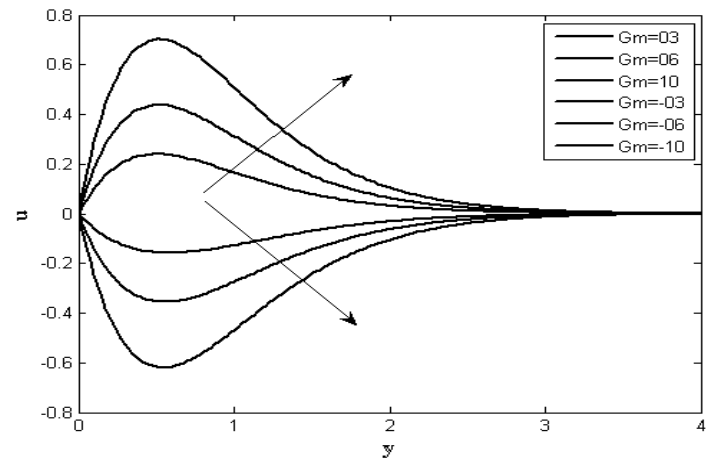

Fig.(3): Velocity profile for different values of Gm

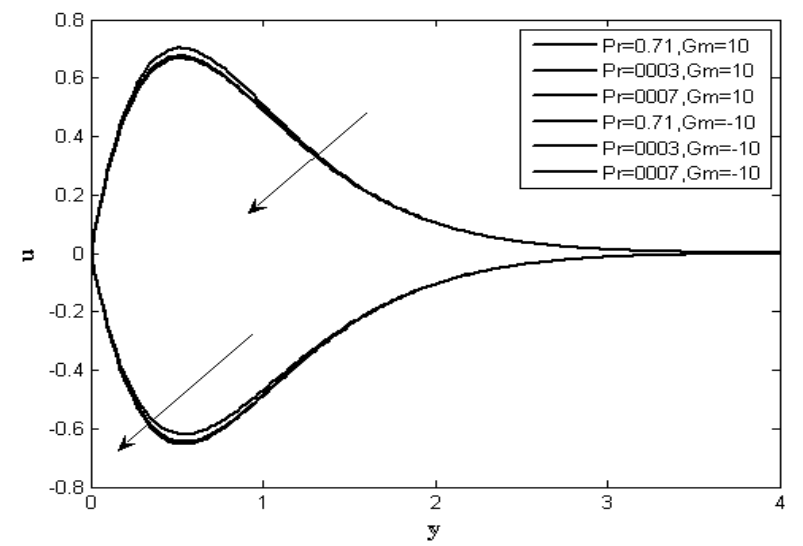

Fig.(4): Velocity profile for different values of $\mathrm{Pr}$

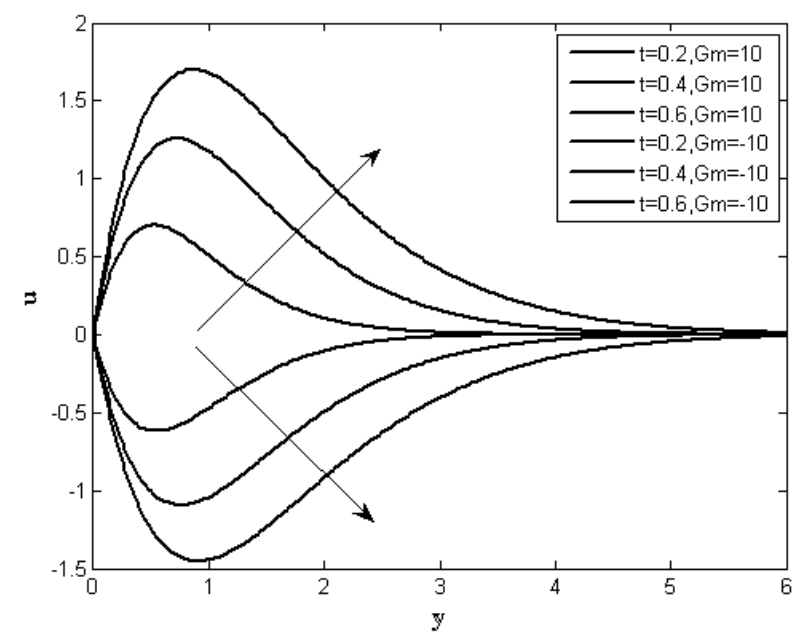

Fig.(5): Velocity profile for different values of $t$ 


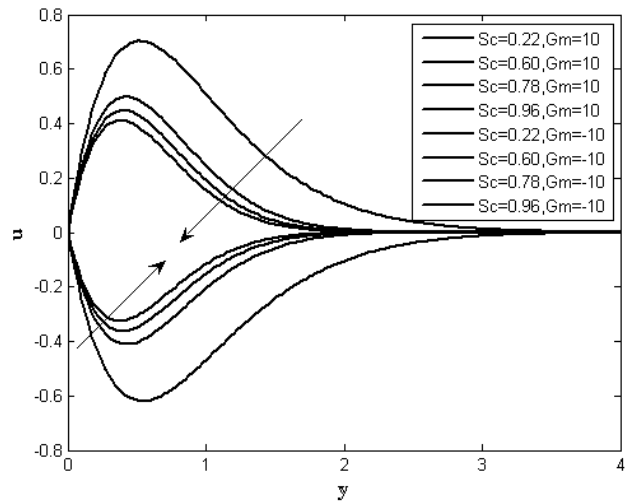

Fig.(6): Velocity profile for different values of Sc

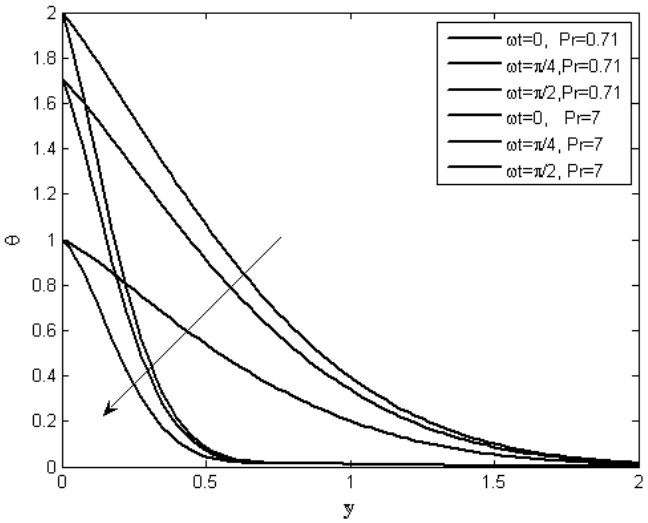

Fig.(7): Temperature profile for different values of $\omega t$

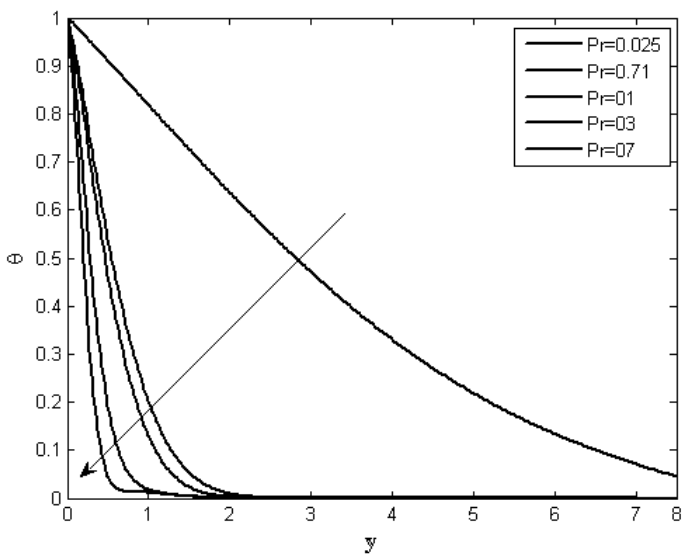

Fig.(8): Temperature profile for different values of $\operatorname{Pr}$

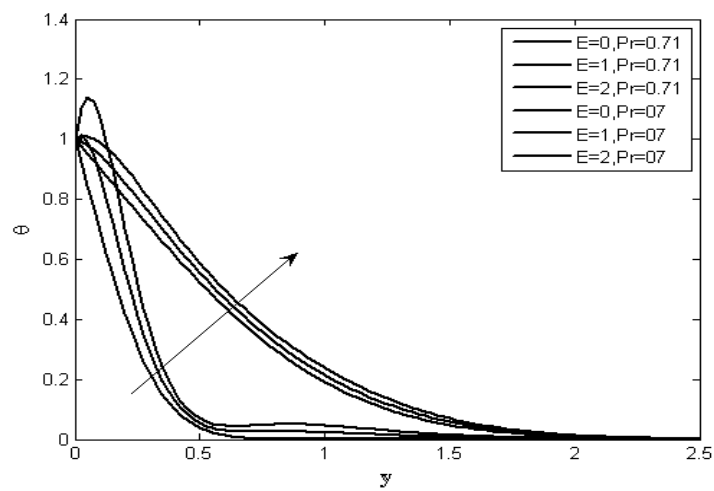

Fig.(9): Temperature profile for different values of $\mathrm{E}$ 


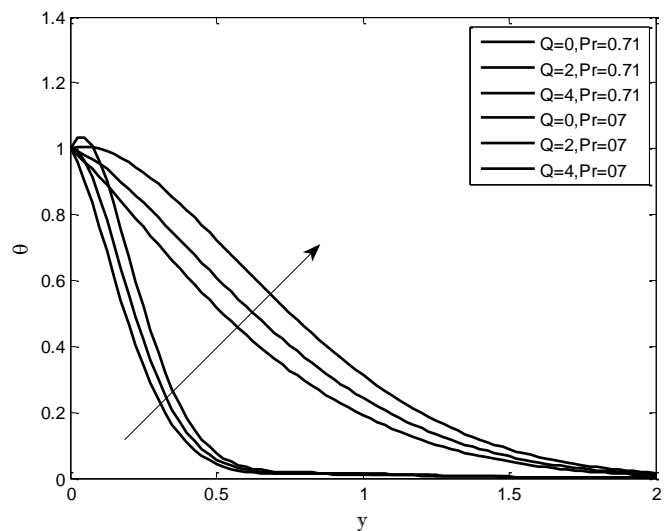

Fig.(10): Temperature profile for different values of $\mathrm{Q}$

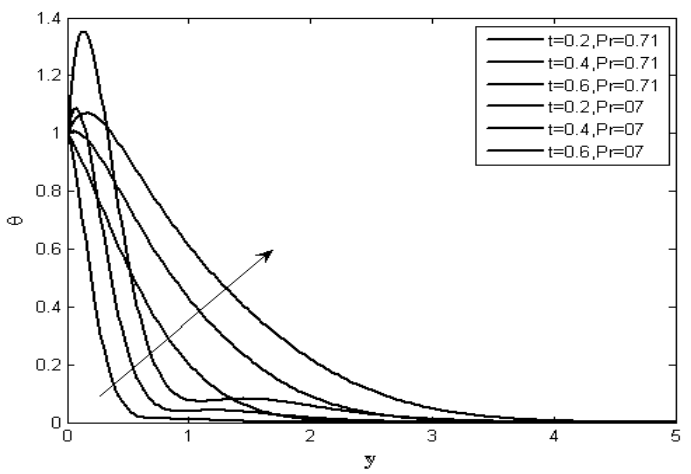

Fig.(11): Temperature profile for different values of $t$

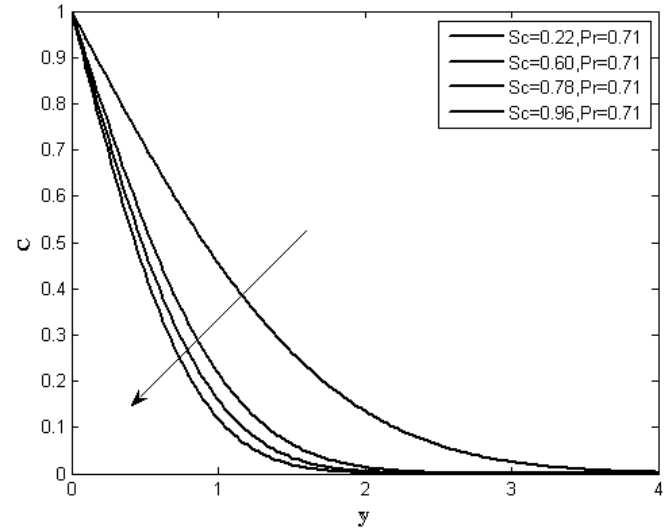

Fig.(12): Concentration profile for different values of Sc

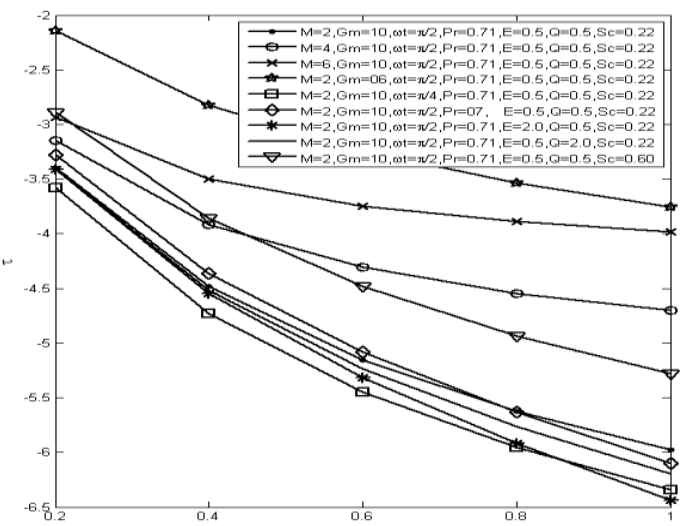

Fig. (13): Skin friction profile 


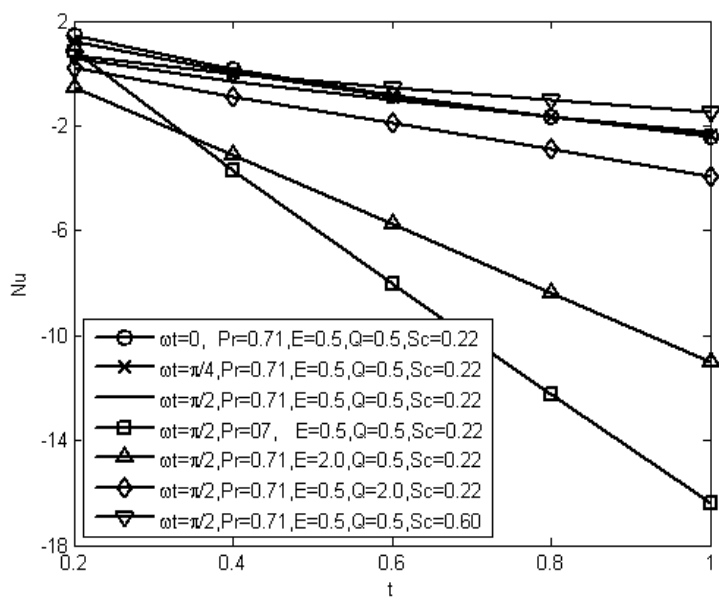

Fig. (14): Nusselt number profile

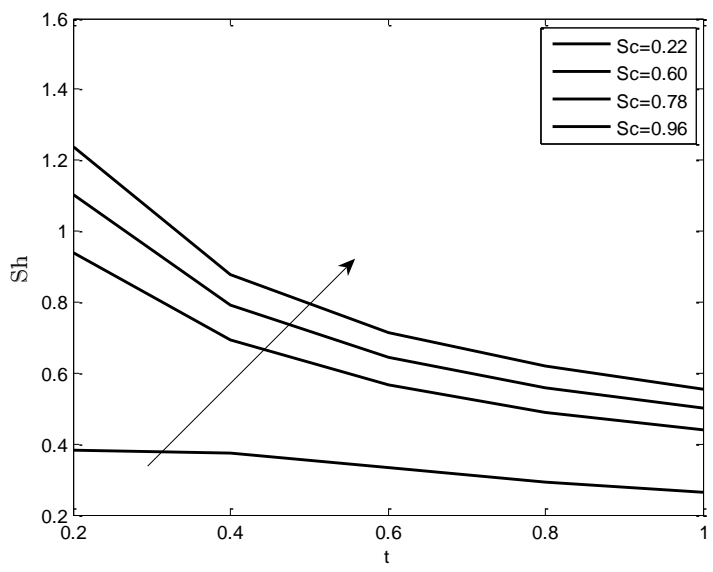

Fig. (15): Sherwood number profile

\section{Conclusions}

This paper presents a numerical study of heat generation effects on the transient hydromagnetic natural convection flow past a vertical plate with mass diffusion and fluctuating temperature about time at the plate, by taking into account the heat due to viscous dissipation. The governing equations are solved by an implicit finite difference method of Crank - Nicolson type. The results are obtained for temperature, velocity, skin - friction, Nusselt number and Sherwood number. The effects of various parameters are discussed on the flow variables and presented by graphs.

From the present numerical investigation, following observations have been drawn:

$>\quad$ Velocity increases and attains its maximum value in the vicinity of the plate and then tends to ' 0 ' as $\mathrm{y} \rightarrow \infty$ due to the variations in phase angle $(\omega \mathrm{t})$, Schmidt number $(\mathrm{Sc})$, time ( $\mathrm{t}$ ), Modified Grashof number $(\mathrm{Gm})$ and Prandtl number (Pr).

$>$ Velocity decreases with an increase in magnetic parameter $(\mathrm{M})$, Schmidt number $(\mathrm{Sc})$ and the reverse effect is noticed for $\mathrm{Gm}<0$. Also Velocity decreases with an increase in phase angle ( $\omega \mathrm{t})$ and Prandtl number (Pr).

$>$ Velocity increases with increasing time ' $\mathrm{t}$ ' and modified Grashof number $(\mathrm{Gm})$ and the reverse effect is noticed for $\mathrm{Gm}<0$.

$>$ The magnitude of temperature is maximum at the plate and then decays to zero asymptotically.

$>$ Temperature falls with an increase in the phase angle $(\omega t)$ and Prandtl number $(\operatorname{Pr})$ for both air and water. The magnitude of temperature for air $(\operatorname{Pr}=0.71)$ is greater than that of water $(\operatorname{Pr}=7)$.

$>\quad$ Temperature enhances due to an increase in the dissipation parameter (E), heat generating parameter $(\mathrm{Q})$ and time ' $t$ ' for both air and water.

$>$ Concentration decreases with an increase in Schmidt number $(\mathrm{Sc})$. 
Skin - friction profile for water is greater than that of air and also it decreases with an increase in Grashof number (Gm), Eckert number (E) and heat generation parameter (Q)' while it increases with an increase in 'magnetic parameter $(\mathrm{M})$, phase angle $(\omega \mathrm{t})$ and Schmidt number $(\mathrm{Sc})$ '.

It is found that the rate of heat transfer falls with increasing $\omega t$, Prandtl number (Pr), Eckert number (E), and heat generation parameter $(\mathrm{Q})$ while it increases with an increase in Schmidt number (Sc).

The rate of concentration transfer increases with increasing values of Schmidt number (Sc).

\section{References}

[1]. Vedhanayagam, M., Altenkirch, R.A. and Eichhorn, R. (1980): A transfor-mation of the boundary layer equations for free convection flow pasta vertical flat plate with rbitrary blowing and wall temperaturevariation, Int. J. Heat Mass Transfer, 23, 12861288.

[2]. Kolar, A.K. and Sastri, V.M. (1988): Free convective transpiration over a vertical plate, a numerical study, Heat and Mass Transfer, 23, 327-336.

[3]. Li Jian, Ingham ,D.B. and Pop, I. (2001): Natural convection from a vertical flat plate with a surface temperature oscillation, Int. J. Heat Mass Transfer, 44, 2311-2322.

[4]. Siegel, R. (1958): Transient free convection from a vertical flat plate, Trans. ASME, 80, 347-359.

[5]. Goldstein, R.J. and Eckert, E.R.G. (1960): The steady and transient free convection boundary layer on a uniformly heated vertical plate, Int. J. Heat Mass Transfer, 1, 208-218.

[6]. Raithby, G.D. and Hollands, K.G.T. (1985): Natural Convection In Hand-book of Heat Transfer Fundamentals,(Rohsenow W.M., Hartnett, J.D. and Ganic, E.N. (eds.)), McGraw-Hill, New York.

[7]. Das, U.N., Deka, R.K. and Soundalgekar, V.M. (1999): Transient free convection flow past an infinite vertical plate with periodic temperature variation, Journal of Heat Transfer, Transactions of the American Society Of Mechanical Engineers, 121, 1091-1094.

[8]. Saeid, Nawaf, H. (2003): Transient free convection from vertical wall with oscillating surface temperature, Asean Journal For Science \& Technology Development, 20, 261-269.

[9]. Lin, H.T. and Yu, W.S. (1995): Combined heat and mass transfer by laminar natural convection flow from a vertical plate, Heat and Mass Transfer, 30, 369-376.

[10]. Mongruel, A., Cloitre, M. and Allain, C. (1996) Scaling of boundary-layer flows driven by double-diffusive convection, Int. J. Heat Mass Transfer, 39, 3899-3910.

[11]. Israel - cookey, C., Ogulu, A., Omubo - Pepple ,V.M. (2003): The influence of viscous dissipation and radiation on unsteady MHD free convection flow past an infinite heated vertical plate in a porous medium with time depedent suction. Int. J. Heat Mass Transfer, 46, 13, $2305-2311$.

[12] Zueco Jordan, J. (2007): Network Simulation Method Applied to Radiation and Dissipation Effects on MHD Unsteady Free Convection over Vertical Porous Plate. Applied Mathematical Modelling, 31, 20, 2019 - 2033.

[13]. Suneetha, S. Bhaskar Reddy, N. Ramachandra Prasad, V. (2008): the thermal radiation effects on MHD free convection flow past an impulsively started vertical plate with variable surface temperature and concentration. Journal of Naval Architecture and Marine engineering, 2, $57-70$.

[14]. Hitesh Kumar (2009): Radiative Heat Transfer with Hydro magnetic flow and viscous dissipation over a stretching surface in the presence of variable heat flux. Thermal Science 13, 2, $163-169$.

[15]. K. Vajravelu and A. Hadjinicolaou, (1993): Heat transfer in a viscous fluid over a stretching sheet with viscous dissipation and internal heat generation, International Communications in Heat and Mass Transfer, 20, 3, 417-430.

[16]. B. Madhusudhana Rao, G.Viswanatha Reddy, M.C.Raju, S.V.K.Varma, (2013): MHD transient free convection and chemically reactive flow past a porous vertical plate with radiation and temperature gradient dependent heat source in slip flow regime. IOSR Journal of Applied Physics, 3, 6, 22-32.

[17]. A. Mamun, Z. R. Chowdhury, M. A. Azim, and M. M. Molla, (2008): MHD-conjugate heat transfer analysis for a vertical flat plate in presence of viscous dissipation and heat generation, International Communications in Heat and Mass Transfer, $35,10,1275-1280$.

[18]. M. A. Azim, A. A.Mamun, andM.M. Rahman,(2010): Viscous Joule heating MHD-conjugate heat transfer for a vertical flat plate in the presence of heat generation, International Communications in Heat and Mass Transfer, 37, 6, 666-674.

[19]. Cowling ,T.G. (1957): Magnetohydrodynamics Interscience Publishers, New York.

[20]. Carnahan, B., H.A. Luther, J.O. Wilkes, (1969): Applied Numerical Methods, John Wiley \& Sons, New York.

\section{NOMENCLATURE}

$u^{\prime} \quad$ velocity component in $x^{\prime}$ - axis

$t^{\prime} \quad$ time

$B_{0} \quad$ the magnetic field component along $y^{\prime}$ - axis

$C^{\prime} \quad$ concentration at any point in the flow field

$D \quad$ mass diffusivity

$C_{p} \quad$ specific heat at constant pressure

$g$ gravitational acceleration

$T^{\prime} \quad$ temperature of the fluid near the plate

$Q^{\prime} \quad$ dimensional heat generating/absorbing parameter

$Q \quad$ dimensionless heat generating/absorbing parameter 
$k \quad$ thermal conductivity of fluid

Pr Prandtl number

Gm modified Grashof number

$M \quad$ magnetic parameter

Sc Schmidt number

$t$ time in dimensionless coordinate

E Eckertnumber

$L_{R} \quad$ reference length

$t_{R} \quad$ reference time

$u$ dimensionless velocity component

$U_{R} \quad$ reference velocity

$C$ dimensionless concentration

\section{GREEK SYMBOLS}

$\beta \quad$ coefficient of volume of expansion

$\beta_{c} \quad$ concentration expansion coefficient

$\rho$ density

$\sigma \quad$ electrical conductivity

$\in \quad$ amplitude (constant)

$v \quad$ kinematic viscosity

$\mu \quad$ viscosity of fluid

$\theta$ dimensionless temperature

$\omega$ frequency of oscillation

\section{SUBSCRIPTS}

$w \quad$ conditions at the wall

$\infty$ conditions in the free stream 